\title{
Swallowable medical devices for diagnosis and surgery: the state of the art
}

\author{
J L Toennies ${ }^{1}$, G Tortora ${ }^{2}$, M Simi ${ }^{2}$, P Valdastri ${ }^{2}$, and R J Webster III ${ }^{1 *}$ \\ ${ }^{1}$ Department of Mechanical Engineering, Vanderbilt University, Nashville, Tennessee, USA \\ ${ }^{2}$ CRIM Lab, Scuola Superiore Sant'Anna, Pisa, Italy
}

The manuscript was received on 2 August 2009 and was accepted after revision for publication on 9 December 2009.

DOI: 10.1243/09544062JMES1879

\begin{abstract}
The first wireless camera pills created a revolutionary new perspective for engineers and physicians, demonstrating for the first time the feasibility of achieving medical objectives deep within the human body from a swallowable, wireless platform. The approximately 10 years since the first camera pill has been a period of great innovation in swallowable medical devices. Many modules and integrated systems have been devised to enable and enhance the diagnostic and even robotic capabilities of capsules working within the gastrointestinal (GI) tract. This article begins by reviewing the motivation and challenges of creating devices to work in the narrow, winding, and often inhospitable GI environment. Then the basic modules of modern swallowable wireless capsular devices are described, and the state of the art in each is discussed. This article is concluded with a perspective on the future potential of swallowable medical devices to enable advanced diagnostics beyond the capability of human visual perception, and even to directly deliver surgical tools and therapy non-invasively to interventional sites deep within the GI tract.
\end{abstract}

Keywords: swallowable devices, capsules, gastrointestinal tract, wireless capsule endoscopy, robotic capsules, minimally invasive surgery, endoluminal devices, surgical endoscopy

\section{INTRODUCTION}

The often repeated assertion that wireless capsule endoscopy (WCE) has rapidly become the preferred method of small intestine diagnosis was underscored recently by the May 2009 announcement of Given Imaging, Inc. (the first commercial producer of camera pills) that over 1 million of their PillCam capsules have been used clinically. PillCams and other commercial capsules (see Fig. 1) have been used to diagnose many diseases including obscure gastrointestinal bleeding (OGIB), cancer, celiac disease, and Crohn's disease, all of which occur in the small intestine [1]. Recently, commercial WCEs have also been extending their reach into other parts of the gastrointestinal (GI) tract including the oesophagus and colon [1]. It is widely agreed that enhancing this technology through the

${ }^{*}$ Corresponding author: Department of Mechanical Engineering, Vanderbilt University, VU Station B 351592, 2301 Vanderbilt Place, Nashville, TN 37235-1592, USA.

email:robert.webster@vanderbilt.edu development of active, robotic capsules that can see, diagnose, and treat will make health care more precise, portable, and personal [2].

Because it requires only the simple and non-invasive swallowing of a pill and no anaesthesia, modern WCE offers an appealing alternative to traditional flexible scope-based endoscopy for visualizing the interior of the GI tract. In this article, only these wireless, swallowable, and pill-like devices are considered. A review of a number of tethered devices is available to the interested reader in reference [3]. Similarly, related technologies that are beyond the scope of this article are those used in natural orifice and transluminal endoscopic surgery (NOTES) devices, as discussed in references $[4]$ to $[8]$. Such devices typically require anaesthesia, which implies low (but non-negligible) risks of cardiopulmonary and other complications [9]. It has also been hypothesized that WCE may, by reducing the indignity and discomfort of endoscopic procedures, have the additional benefit of encouraging many who currently forgo medically recommended examinations (particularly colonoscopy) to have them done [10]. 


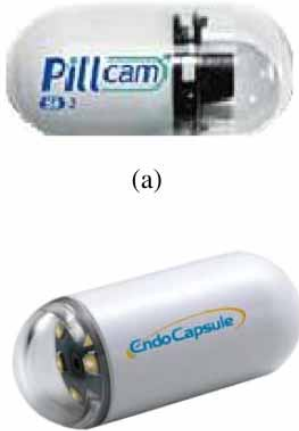

(d)

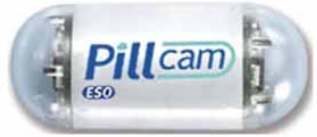

(b)

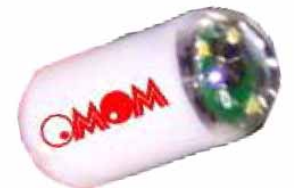

(e)

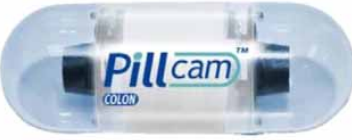

(c)

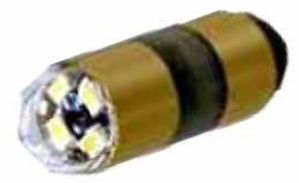

(f)

Fig. 1 Current commercially available swallowable camera capsules include (a) PillCam SB2, (b) PillCam ESO, (c) PillCam Colon, all by Given Imaging, Inc., as well as (d) EndoCapsule of Olympus, Inc., (e) OMOM of Jinshan Science and Technology Group, and (f) MiRo of Intromedic Co. These capsules carry cameras into the GI tract for visual inspection. While most of the capsules shown are designed for the small intestine, PillCam ESO is an oesophageal capsule and the PillCam Colon carries two onboard cameras for use in the large intestine. All capsules are $11 \mathrm{~mm}$ in diameter $\times 26 \mathrm{~mm}$ in length except for OMOM $(13 \mathrm{~mm}$ $\times 27.9 \mathrm{~mm})$, MiRo $(10.8 \mathrm{~mm} \times 24 \mathrm{~mm})$ [14], and PillCam Colon $(11 \mathrm{~mm} \times 31 \mathrm{~mm})$ [15]

Robotic systems including sensors and actuators have shown great promise in extending the interventional and diagnostic capabilities of WCEs, and the purpose of this article is to review the state of the art in such systems. Because a number of recent and thorough reviews of commercial passive (imaging-only) camera pills are already available to the interested reader (see references $[\mathbf{1}],[\mathbf{3}],[\mathbf{1 1}]$, and [12]), the present authors treat them only briefly here, providing a complementary review of recent robotic advancements in swallowable sensors and actuators, and the associated electronics, telemetry, and power systems that enable them to work. A brief survey of some (but not all) of these topics has been given in reference [13], and in the current paper an expanded review of all aspects of state-of-the-art WCE technology is provided.

The sections that follow first discuss the unique requirements of the various areas of the GI tract and briefly treat commercial capsules. Then, a high-level overview of the modules that can be included in a robotic WCE is given, after which each module is discussed in depth. The article concludes in section 10 with a perspective on the role robotic technologies can be expected to play in the future of WCE.

\section{CAPSULE ENVIRONMENT AND MODULES}

The GI tract consists of oesophagus, stomach, small intestine, and large intestine (or colon), each of which presents different challenges for WCEs (see Fig. 2). The oesophagus, which connects the throat to the stomach, is a hollow, muscular tube that for an average adult is approximately $25 \mathrm{~cm}$ long, and $1.5-2 \mathrm{~cm}$ in diameter [16]. Oesophageal capsules (e.g. the PillCam

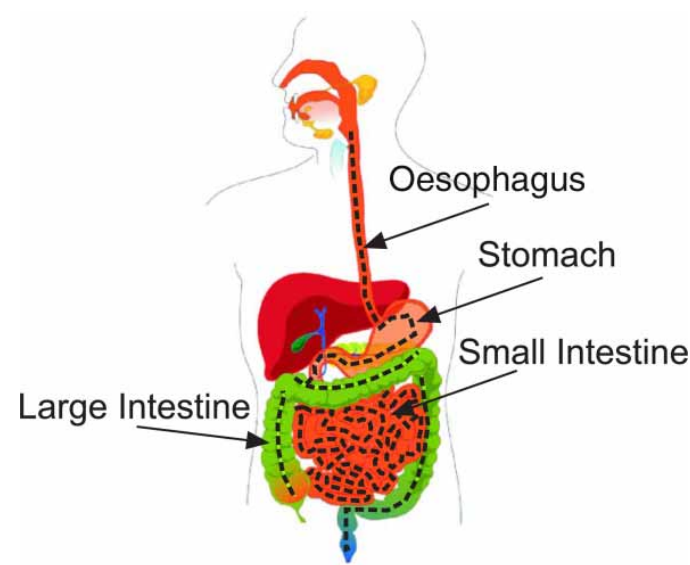

Fig. 2 A Schematic of the GI tract showing the oesophagus, stomach, small intestine, and large intestine (or colon), each of which present a unique set of challenges for WCEs. The typical path of a WCE is shown by the dashed black line. Note that the orientation of a capsule along this path is arbitrary due to the lack of active locomotion

ESO and ESO2 by Given Imaging, Inc.) require high frame rates due to the rapid rate of progression (complete transit in approximately $10 \mathrm{~s}$ [16]) of the capsule within the oesophagus. Fortunately, since such capsules only need to function for a few minutes, battery life is a much lesser concern than in other areas of the GI tract [12]. Oesophageal capsules can also be used for longer-term monitoring when combined with a braking or anchoring mechanism such as legs $[\mathbf{1 7}, \mathbf{1 8}]$ or magnets [19] (which are discussed further in section 7). This is particularly useful in diagnosis of gastro oesophageal reflux disease (GERD), which requires at least 24 hours of $\mathrm{pH}$ monitoring. 
The Medtronic Bravo Capsule, which does not collect images, is designed for this purpose but requires fixation to the oesophageal wall via an endoscopic procedure [20].

After passing through the oesophagus, the capsule reaches the stomach, a large, typically deflated elastic sac that is lined with a thick mucous membrane and contains gastric juices. In its deflated state, the stomach contains many longitudinal folds, and at the pylorus, the narrowest part of the stomach, it is about $2 \mathrm{~cm}$ in diameter [16]. Owing to the challenge of maintaining traction on the stomach wall, few capsules have been purposely designed for this portion of the GI tract, although prototypes have been developed for a propeller-driven 'swimming' capsule [21] (see section 7) and a multi-module assemblable and reconfigurable robotic system consisting of several units intended to be independently swallowed [22].

A capsule exits the stomach into the small intestine, where WCEs have made their most significant clinical impact. The small intestine is an elastic lumen, $3-4 \mathrm{~cm}$ in diameter, and is the longest portion of the GI tract (approximately $670-760 \mathrm{~cm}$ in length). This tubular structure, which winds and curves many times in the abdomen, has villi coating its internal surface. It normally contains semi-digested foods, pancreatic juices, enzymes, and bile [16], although the food products are typically removed via ingestible agents prior to capsule endoscopy. WCEs are pushed through the small intestine via peristalsis (the natural muscle activity that normally moves decomposing food products). Peristalsis generally occurs over short segments and propagates at a rate of $1-2 \mathrm{~cm} / \mathrm{min}$ [16]. Commercial capsules designed for the small intestine (see Fig. 1) include the PillCam SB and SB2 and Agile Patency System (a self-disintegrating pill [12]) by Given Imaging, Inc., the EndoCapsule by Olympus, Inc., the OMOM capsule by the Jinshan Science and Technology Group Co., Ltd., and the MiRo capsule by Intromedic Co. The Agile capsule, an accessory to the PillCam, can be used prior to the ingestion of the PillCam SB to verify adequate capsule passage through the GI tract in patients with known or suspected strictures. This capsule assists in detecting the problem of capsule retention, which occurs in 0.75 per cent of OGIB cases, but may occur at a rate of up to 6.7 per cent in patients with Crohn's disease [12]. While each of these commercial capsules is designed for use in the small intestine, they vary in the optical sensors (complementary metal oxide silicone (CMOS) or charged couple device (CCD)) used, their size, and their attainable frame rates. See section 5 for further information as well as reference [14] for an in-depth review of commercial camera capsule capabilities.

The last region of the GI tract encountered by a WCE is the large intestine or colon, which measures about $150 \mathrm{~cm}$ in length and $6 \mathrm{~cm}$ in diameter $[\mathbf{1 6}, \mathbf{2 3}]$. While it does not contain as many folds as the small intestine,

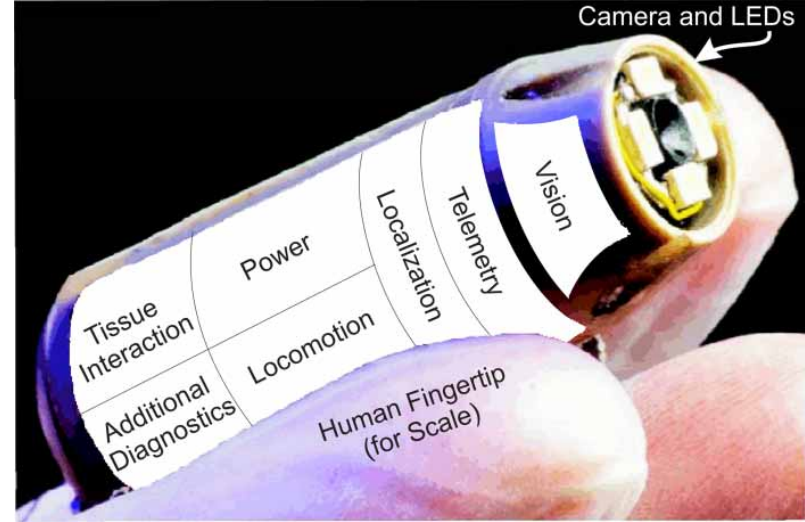

Fig. 3 Schematic diagram of possible modules in a robotic wireless capsule

the large intestine contains two flexures (corners) that a capsule must navigate around. The larger lumen makes diagnosis difficult for capsules designed for the small intestine because they tumble in the larger lumen and generally cannot view the entire internal surface. A number of devices have been designed to work in the colon by entering through the rectum and travelling against peristalsis (which is the approach of standard colonoscopy) as reviewed in reference [3]. WCEs designed for the colon include PillCam Colon by Given Imaging, Inc. and the legged capsule of Valdastri et al. [10]. The time of passage of a capsule through the entire GI tract is generally about 8-10 h, with approximately $1 \mathrm{~h}$ spent in the stomach, $4 \mathrm{~h}$ in the small intestine, and $5 \mathrm{~h}$ in the colon [24].

\subsection{Robotic endoscopic capsule modules}

There are a number of modules possible in a robotic WCE (see Fig. 3), although most capsules built to date include only a subset of these due to space constraints. Whether it will ultimately be possible to create a single robotic device with all of these modules that can both diagnose and treat disease, or whether a group of task-specific capsules will work cooperatively (a more likely initial scenario), a large number of robotic modules will be required. The major modules of a robotic capsule system are localization, telemetry, vision, non-visual sensors, locomotion, interventional systems, and power. Each of these modules individually poses a variety of worthy engineering challenges, and integrating two or more of them is also challenging. Subsequent sections of this article discuss the technical progress that has been made in each of the general modules.

\section{LOCALIZATION}

To determine the location of lesions and other structures visualized in capsule images (for future 
follow-up interventions or to enhance diagnosis) systems must be developed to measure the position and orientation of the capsule when images are collected. Useful spatial information includes the capsule's position in three-dimensional (3D) space, the distance it has travelled along the GI tract, and the region of the GI in which it is located. A number of methods for obtaining localization information have been explored in feasibility studies, including systems based on radio frequency $(\mathrm{RF})$ triangulation, magnetic tracking, computer vision, and ultrasound, as described below.

3D position information can be obtained by both $\mathrm{RF}$ triangulation and magnetic tracking. RF triangulation systems typically use an external sensor array that measures signal strength of capsule transmissions at multiple points and uses this information to estimate distance. This type of localization method is used in PillCam SB systems [25]. However, the noisy nature of distance estimates based on RF signal strength resulted in an average experimental error of $37.7 \mathrm{~mm}$ [26] in one study. A second approach to obtain 3D position information is magnetic tracking of a permanent magnet that is placed inside the capsule and detected by a skin-mounted magnetoresistive sensor array [26]. Using both field strength and field direction, a calibrated sensor array can achieve an average position error of $3.3 \mathrm{~mm}$ and an average orientation error of $3^{\circ}$ [27]. This system uses a static magnetic field and can collect measurements at $3.33-5 \mathrm{~Hz}$. Another 3D magnetic detection system called 3D-MAGMA (Innovent Technology, Germany), collects individual measurements at $50 \mathrm{~Hz}$, before filtering and processing to provide accurate position information at a rate of $2 \mathrm{~Hz}$. It uses 27 sensors, achieving an accuracy of $5 \mathrm{~mm}$ and $2^{\circ}[\mathbf{2 8}]$, and also uses magnetic information to estimate velocity.

Distance along the GI tract and determination of the GI structure in which the capsule is located have been approached through computer vision. While 'dead reckoning' of distance along the intestine may seem like an obvious approach for deducing capsule distance travelled, it is not straightforward. Commercial (non-oesophageal) capsules capture images at only 2$4 \mathrm{~Hz}$ (see section 5), and their motion is erratic (i.e. they may spend relatively long periods of time stationary before moving forward rapidly a short distance, and they may even flip over, aiming their camera back up the lumen from where they came). Furthermore, there are few distinctive features on the wall of the GI tract for a computer vision algorithm to track. However, these difficulties have not prevented noteworthy attempts at computer vision approaches to capsule localization. One recent approach uses moving picture experts group (MPEG)-7 image features, vector quantization, principal component analysis, and neural networks to classify images as belonging to the upper or lower (i.e. the colon) GI tract and achieves 95 per cent accuracy [29]. Another computer vision method uses an energy-based event boundary detection algorithm to identify different digestive organs based on their unique patterns of muscular contraction, correctly classifying events with 76 per cent accuracy $[\mathbf{3 0}]$.

Inertial sensing based on accelerometer data has also been used for capsule localization [31]. In this work, a $3 \mathrm{~mm} \times 3 \mathrm{~mm}$ digital triaxial accelerometer, which operates at $20 \mathrm{~Hz}$, was integrated within the capsule and data were transmitted over ZigBee technology (see section 4) to an external computer. This system successfully estimated orientation with $6^{\circ}$ accuracy. It should also be noted that since acceleration is directly measured, velocity can be obtained more accurately than position because it requires only a single integration. Another possible localization approach is the use of ultrasonic pulses emitted from outside the body and echoed by the capsule [32], although this has not yet been directly tested in the GI tract. The InteliSite and Enterion drug delivery capsules (see section 8 for more information) use Gamma Scintigraphy as a means of localization. This method requires a radioactive agent be placed inside the capsule so that it can be tracked using gamma cameras [33]. A recent review of patent literature by Moglia et al. [34] also discusses the possibility of combining magnetics and X-ray imaging to localize a capsule [35].

\subsection{Localization summary and challenges}

Since the GI tract is a long tubular structure that folds upon itself many times and is free to move within the abdominal cavity, it is a challenging environment in which to localize capsule position. Furthermore, the GI tract lacks straightforward landmarks, making discernment of capsule location from image information difficult even for trained physicians. However, identifying the physical location of capture of each capsule image is important in both diagnostic and therapeutic applications of WCEs. This has lead to a number of innovative approaches to the localization problem, as reviewed above. However, these systems are currently at the proof of concept stage, and it remains unclear which will be the most useful and easily implementable, while providing sufficiently accurate information, in future clinical WCE applications.

\section{TELEMETRY}

Endoscopic capsules rely heavily on wireless communication to transmit images and receive commands. The two most important metrics defining the quality of capsule wireless telemetry are power efficiency and data rate. Some capsules employ commercially available telemetry chips, while others utilize custom purpose-built solutions, see reference [36] for an overview. Given Imaging, Inc., for example, uses a 
custom telemetry chip produced specifically for their PillCams, made by Zarlink, Inc. The power consumption of this chip is $5.2 \mathrm{~mW}$, and it has a data rate of 2.7 Mbps with a carrier frequency of 403-434 MHz [36]. This telemetry link is unidirectional and is used for transmitting images out of the capsule only. Other custom unidirectional modules for image transmission also exist. The system by Shen et al. [37] transmits on a $416 \mathrm{MHz}$ carrier frequency at slightly lower power levels $(4 \mathrm{~mW})$ and data rates (2 Mbps) than the Zarlink chips described above. Thone et al. [36] developed a similar design that transmits at $2 \mathrm{Mbps}$ at only $2 \mathrm{~mW}$ of power consumption, using a $144 \mathrm{MHz}$ carrier frequency.

Bidirectional data transmission is often essential for robotic capsules, which must receive and act upon commands sent to them. ZigBee, which uses the IEEE 802.15.4 standard, has been applied for this purpose in proof of concept experiments by Valdastri et al. [39]. Developed with home and building automation, industrial control and monitoring, wireless sensor networks, and consumer electronics in mind, ZigBee is characterized by low power, low data rate, long battery lifetime, and high networking capability. The data rate of ZigBee is $250 \mathrm{kbps}$, which is sufficient for bidirectional transmission of control signals, but not for images. An architecture applying ZigBee together with a commercial microcontroller (CC2430 from Texas Instruments) for capsular endoscopy has been developed by Susilo et al. [38] (see Fig. 4).

An innovative new method of information transmission known as electric-field propagation exploits the human body as a conductive medium for data transmission. This has been applied in the new commercial MiRo capsule by Intromedic Co. While power levels and data rates have not yet been reported, it is hypothesized that this method may offer higher data rates with lower power consumption than existing

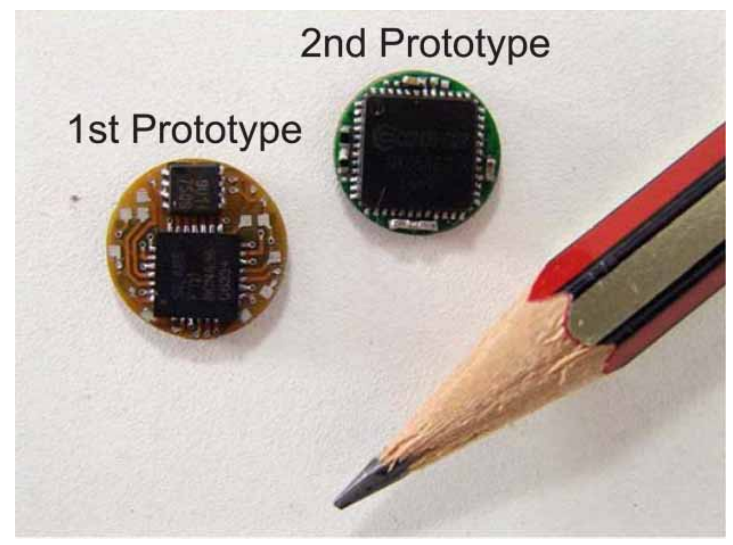

Fig. 4 Custom miniature telemetry and control circuit boards developed by Susilo et al. [38], which utilize ZigBee and a commercial microcontroller for communication and control of a WCE
RF technologies due to the elimination of powerconsuming RF transmission components [40].

\subsection{Telemetry summary and challenges}

Data rates are limited in current unidirectional telemetry links for video signals, which limit current image transmission frequency to $2-4 \mathrm{~Hz}$ (see section 5). Paths forward towards more rapid imaging include improved image compression algorithms (see section 5) and/or enhanced telemetry link technology, with electric-field propagation being the most promising current technology. In designing a telemetry link, one must be concerned with not only keeping electrical power consumption low and data rates high, but also maintaining transmission signal power within medically safe limits (see reference [39]) without sacrificing transmission link reliability. Robotic devices often require bidirectional communication, and ZigBee is the most promising current technology that has been used in this application.

\section{VISION}

Capsular endoscopy owes its very existence to the miniaturization and quality enhancements of cameras that have taken place in the past several years [41], which enabled the first camera pills. The goal of all current capsule vision systems is to use onboard components to obtain clear images of areas of interest in the GI tract and to transmit those images efficiently to an offboard platform for manual analysis by a physician or automated analysis by a computer algorithm. Vision system enhancements are an active area of WCE research, and efforts are underway towards miniaturization, efficiency enhancements, improving image quality and information content, and methods of differentiating the few diagnostically useful images from the many total images returned from the capsule. Current commercial and research capsules and capsule robots typically make use of miniature CMOS $[40,42,43]$ and CCD $[44]$ imaging chips, and a detailed review of their vision capabilities can be found in reference [1]. Briefly, there exists single (PillCam SB, Given Imaging, Inc.) and multi-lens (PillCam SB2) solutions, with four (PillCam SB) to six (Olympus EndoCapsule) light emitting diodes (LEDs), viewing angles from $140^{\circ}$ (PillCam SB) to $156^{\circ}$ (PillCam SB2), and data rates of $2 \mathrm{~Hz}$ (PillCam, OMOM and Olympus capsules) to $3 \mathrm{~Hz}$ (MiRo, Intromedic Co.). The PillCam SB, OMOM, and MiRo store all images for later viewing, while the PillCam SB2 and the Olympus EndoCapsule provide the capability of viewing images as they are acquired. The oesophageal capsules (PillCam ESO and ESO2) require higher data rates for shorter durations and include two cameras capturing at up to 18 images per second with a battery life of 20-30 min [42]. The lone example of 
a commercial capsule designed for the colon (PillCam Colon) also has two cameras capturing images at $4 \mathrm{~Hz}$ each. Because it can be switched off until the capsule reaches the colon, the device has a battery life quoted at $10 \mathrm{~h} \mathrm{[1]}$, but its active period is shorter.

\subsection{Image acquisition hardware}

To obtain clear images, modern endoscopic capsules rely on onboard image acquisition hardware including a vision sensor, lens, illumination source, and image compression chip. These combine to deliver images (see Fig. 5 for an example), the quality of which can be described by metrics for colour resolution, spatial resolution, and frame rate [45]. As mentioned above, most image acquisition chips are currently based on CMOS technology, with the exception of the Olympus EndoCapsule, which uses CCD. When compared with the PillCam SB, it has been shown that the EndoCapsule generally scores higher in image quality and resolution, but both yield comparable diagnostic performance $[46,47]$. CMOS technology has also been applied to a variety of robotic capsules within the Versatile Endoscopic Capsule for Gastrointestinal Tumor Recognition and Therapy (VECTOR) European project, where a monolithic $320 \times 240$ active-pixel colour-RGB or grey level camera-on-a-chip sensor was developed and tested in the ex-vivo setting $[\mathbf{4 8}, 49]$. There has also been some recent work aimed at combining ideas from CMOS and CCD chips to create improved hybrid cameras [41].

Optics are also important for high-quality vision, and there has been some work towards developing autofocus lenses. A prototype system by Cavallotti et al. [14] uses a liquid lens actuated by a pulse width modulated signal to adjust focal length from 30 to $100 \mathrm{~mm}$. Good illumination is also essential for

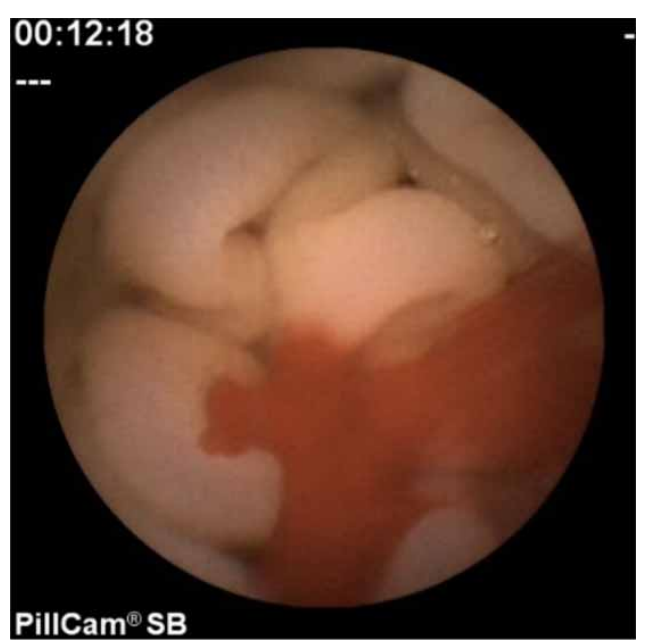

Fig. 5 View of a bleeding lesion in the small intestine, captured by a PillCam SB (image courtesy of Rajesh Kumar, Johns Hopkins University) high-quality imaging and can even yield enhanced information beyond human visual capacity. Promising techniques include chromoendoscopy and autofluorescence (light working in conjunction with dyes and chemicals applied to the intestinal lining), confocal laser endomicroscopy (for real-time histology), and spectroscopy (which has been used to detect dysplasia) [50]. Endoscopic capsules may in the future serve as a platform for carrying many of these and future optical diagnosis techniques deep into the GI tract.

\subsection{Image compression and software}

Image compression is required for rapid image transmission over the relatively low data rate telemetry links described in section 4 . The images collected from capsule-based cameras generally contain a large amount of information (a VGA image with 8-bit colour information contains $2.45 \times 10^{6}$ bits, and many current capsules capture at $1 / 4$ this resolution) before compression. Since capsules have limited power, compression must be efficient both in terms of electrical power and time, while retaining the essential information stored in the image. The exact level of compression necessary depends on the application and the medical objective of the capsule. While current PillCams produce diagnostically useful results, there is little debate that higher frame rates would be desirable. For future robotic capsules that may be teleoperated by a physician, it is likely that frame rates of 20 or more will be required $[\mathbf{5 1}]$.

Some capsule image compression algorithms process the images acquired in two steps: demosaicking and transforming from RGB space to $\mathrm{YCbCr}$ space, before quantizing the image for compression [52]. One example that uses this standard compression technique, but implements a prediction algorithm as its underlying principle, can obtain compression ratios of 20. Using this technique, a $4 \mathrm{~mm}^{2}$ compression chip that consumes only $7.5 \mathrm{~mW}$ has been fabricated [51] Another low-powered image compressor developed by Lin et al. skips the preprocessing steps of demosaicking and colour-space transformation by starting with the raw images in Bayer pattern format and then processing the R, G1, G2, and B signals separately. This technique reduces the computation load required to compress images by 75 per cent at $14.92 \mathrm{~mW}$ of power [52]. When compressing images, it is also important to conserve memory allocation that requires space onboard the capsule [51]. One approach that specifically addresses this uses a Bayer colour filter array to achieve performance comparable to a more complex wavelet-based coder, while maintaining low power consumption [53].

Advancements have also been made in software for onboard or offboard analysis of capsule images. 
Onboard software can enable closed-loop control of camera orientation for improving image quality [54]. It can also be used for in-situ capsule video analysis in order to detect and predict upcoming video images that may be of concern to a clinician [55]. External postprocessing software for capsule images is also extremely useful because a typical small intestine capsule will return approximately 50000 total images from a single use [56], but only a very small fraction of these images will contain useful diagnostic information. For this reason, algorithms are under development to sort these images and guide the physician to the most important frames, with the future intent of aiding the physician by automatically suggesting appropriate diagnoses for lesions identified [56].

\subsection{Vision summary and challenges}

The challenges in vision involve obtaining clear, sharp images of diagnostically useful areas of the GI tract and compressing them sufficiently (with minimal information loss) for fast transmission over the telemetry link. While the current performance of capsule-based cameras (typically $2-4 \mathrm{~Hz}$ at $1 / 4 \mathrm{VGA}$ resolution) is good enough for some diagnostic tasks and perhaps for some future automated robotic tasks that do not involve real-time human interaction, there is little debate that speed and resolution improvements would be desirable. Such improvements will be necessary to enable some future teleoperated robotic capsules envisioned by researchers, which will require imaging at approximately $24 \mathrm{fps}$ so that the images returned have the appearance of video to the human operator. Unless there are dramatic breakthroughs in telemetry, advancements towards this goal are likely to include increasingly sophisticated image compression algorithms. It is also important to develop automatic image analysis algorithms in both the onboard and offboard contexts, to assist with real-time control and identification of the few diagnostically useful images among the thousands typically returned, respectively.

\section{DIAGNOSTIC SYSTEMS BEYOND IMAGE ACQUISITION}

It is in principle possible to carry a wide range of biosensors onboard a capsule. Quantities that have been measured to date by capsule-based sensors include $\mathrm{pH}$, pressure, oxygenation, electrical conductivity, temperature, and blood detection. An example of a capsule with integrated pressure, temperature, and $\mathrm{pH}$ sensing capabilities is presented in reference $[\mathbf{4 5}]$.

$\mathrm{pH}$ sensing can detect acidic reflux, and impedance monitoring can detect non-acidic reflux, both of which are useful diagnostically. Gonzalez-Guillaumin et al. [19] developed a swallowable capsule that can perform

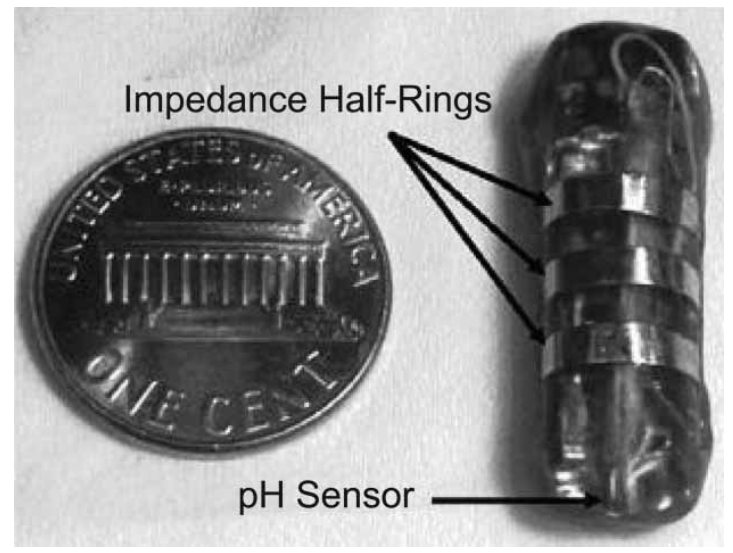

Fig. 6 Prototype of the $\mathrm{pH}$-sensing capsule that uses a voltage difference to measure $\mathrm{pH}$ and half-ring electrodes for impedance sensing (@2007 IEEE) [19]

both of these tasks, as shown in Fig. 6, providing 24-h monitoring for the detection and evaluation of GERD. One noteworthy aspect of this design is its ability to be held to the oesophageal wall through an external magnet placed near the throat. This may be an advantage over the Medtronic Bravo Capsule, which is designed for the same clinical objective but requires thin metal spikes for attachment to the oesophageal wall [20].

Temperatures can be measured in the GI tract by the VitalSense ingestible telemetric physiological monitoring system, that uses thermistor-based sensing, as well as the CorTemp pill system that uses the frequency of oscillation of a crystal for temperature sensing [57]. Other $\mathrm{pH}$ and temperature measurement capsules include the Philips iPill (also discussed in section 8) $[\mathbf{8}, \mathbf{5 8}]$ and a design by van der Schaar et al. that can also expel a small amount of liquid [34]. Two other sensing capsules reviewed in reference [13] include the Lab-on-a-Pill capsule [59] which can sense $\mathrm{pH}$, conductivity, and oxygen levels, and a patented design known as the Stanford endocapsule [60] that alludes to optical, chemical, temperature, and $\mathrm{pH}$ sensing capabilities. Moglia et al. [34] also review other recent patents on capsule-based biosensors.

There has also been research towards detecting blood in the intestine and stomach via microsensors. Researchers from the VECTOR project, in collaboration with novineon Healthcare Technology Partners $\mathrm{GmbH}$ and Sensocor Ltd. developed a system consisting of a bleeding detection capsule (ingestible and implantable versions) that communicates with an external event monitor. LED's onboard the capsule emit red and blue light, identifying the presence of blood by transmission spectroscopy.

Another diagnostic capability that would be useful onboard a capsule is optical biopsy. Standard biopsy in the colon currently requires ten relatively large (2$3 \mathrm{~mm}^{3}$ ) tissue samples to be taken from the same location and analysed within $1-2 \mathrm{~h}$ of extraction, which are 
challenging (and perhaps impossible) requirements for a mobile swallowable capsule. Optical biopsy provides a potential alternative using the properties of light for diagnosis. Promising imaging techniques for optical biopsy include fluorescence endoscopy, optical coherence tomography, confocal microendoscopy, light-scattering spectroscopy, raman spectroscopy, and molecular imaging, all of which are discussed in detail in reference $[\mathbf{6 1}]$.

\subsection{Sensing summary and challenges}

Significant challenges in integrating sensing onboard WCEs are in developing and miniaturizing the biosensors themselves. Additional challenges arise in packaging them onboard the capsule where they must be shielded from unwanted environmental contact and exposed when desired. As with all other capsule components, sensors must also be power efficient and able to be integrated with other modules. A number of promising sensing methods have been developed including sensors for pressure, temperature, electrical conductivity, and $\mathrm{pH}$, and optical biopsy techniques hold great promise for extending the diagnostic capabilities of endoscopic capsules.

\section{LOCOMOTION}

Current commercial capsules move through the GI tract by means of peristalsis alone. However, such movement is unpredictable and inconsistent, which results in incomplete evaluations approximately 20 per cent of the time [62]. Performance is even worse in the large intestine, where the sensitivity of capsule endoscopes for detecting colonic lesions is low compared with the use of conventional colonoscopy [15]. Active locomotion has the potential to improve capsule imaging consistency and coverage, by enabling the capsule to suspend itself in the middle of the lumen and move forward, backward, and to stop in place at areas of interest [10]. Two main approaches have been developed for WCE locomotion: (a) internal and (b) external. Internal locomotion is defined as the use of micromechanisms integrated solely onboard the capsule platform for locomotion. In contrast, external locomotion utilizes propulsive forces transmitted by an external system, typically a magnetic field.

\subsection{Internal locomotion}

Advantages of internal locomotion are the ability to obtain precise motion relative to the lumen due to local contact, and the fact that no external magnetic field generators are required. Another advantage of some mechanisms (such as legs) is the ability to simultaneously distend tissue away from the camera lens
[10]. The major drawback is the need to supply relatively large actuators with a significant amount of power. A number of different internal locomotion strategies have been developed over the past few years, as discussed below.

Vibratory actuation can aid the forward progression of a capsule along the GI tract by reducing friction with its environment [63]. Here, the capsule contains a micromotor with an assymetric mass on the rotor to create vibrations around its central axis. The advantage of this method of locomotion is its mechanical simplicity. Challenges include lack of active orientation control and difficulty obtaining good images during vibration. Furthermore, the capsule cannot actively hold a given position or reverse direction and travel back up the intestine.

Another means of locomotion inspired by biology that has been applied to WCEs is earthworm-like motion. Kim and colleagues [64] have developed two prototypes, one $13 \mathrm{~mm}$ in diameter by $33 \mathrm{~mm}$ in length, and another $15 \mathrm{~mm}$ in diameter by $30 \mathrm{~mm}$ in length $[65,66]$, as shown in Fig. 7 (left). The first device propels itself by cyclic compression/extension of shape memory alloy (SMA) spring actuators, while alternately anchoring its ends to a surface using directional spines (micro needles) mounted at each end. The locomotion method of the second prototype is the same as the first, but it consists of two bellows joined by a custom impact-based piezo actuator. Both designs were tested in ex-vivo porcine intestine and achieved velocities of $0.85 \mathrm{~cm} / \mathrm{min}$ (one point of contact) and $1.47 \mathrm{~cm} / \mathrm{min}$ (three points of contact) for the first prototype, and $13.38 \mathrm{~cm} / \mathrm{min}$ with an $11 \mathrm{~mm}$ stroke for the second prototype. The first prototype moved slower than the second due to the time constants associated with heating and cooling SMA actuators. Directional spines provide the advantage of a passive anchoring mechanism that does not require power or additional actuators, but likely at the expense of precluding bidirectional motion.

Another bio-inspired solution is a cilia system for locomotion [67]. The $15 \mathrm{~mm}$ diameter by $35 \mathrm{~mm}$ long capsule contains six units (intended to mimic biological cells), with two appendages that extend from each unit (mimicking cilia extensions). Each unit is driven by two SMA actuators to enable bidirectional motion at a maximum velocity of $2.4 \mathrm{~cm} / \mathrm{min}$. However, the power consumption of this design is currently high due to numerous SMA actuators. In order to increase friction with the intestinal wall, design optimization of the leg-like cilia extensions has also been performed [68].

It is also possible to produce locomotion in the GI tract using a 'paddling' technique where leg-like fins travel the length of the capsule [69]. At the back of the capsule, the fins retract before recycling to the front of the capsule for the next paddle stroke. An advantage of the paddling motion is the rapid velocity it enables of up to $18-36 \mathrm{~cm} / \mathrm{min}$ depending on the number of 
curves in the section of the GI it traverses, although it does not appear to be able to achieve bidirectional motion in its current form.

Including legs on a capsule has been found useful for both anchoring and locomotion in the GI tract. A robotic capsule with an anchoring mechanism designed for the oesophageal region of the GI tract has been developed by Tognarelli et al. [18]. The design uses three nitinol flexible legs with compliant feet that deploy when released by a motor and can be retracted again when desired. A design similar in intent though employing a different anchoring foot design is a capsule that uses legs with bio-inspired feet that enhance adhesion to the oesophageal wall [17]. This design is envisioned as a follow-up device to initial diagnosis and localization of pathology by current commercial capsules for somewhat longerterm or higher image quality monitoring. Extending the idea of this anchoring mechanism using micropatterned adhesives, Karagozler et al. [70] combine two of the anchoring modules together to develop a locomotion module for an endoscopic capsule. This capsule has six legs, three on each end, that are actuated by coil-type SMA wire in an alternating fashion to mimic a crawling motion. As in models discussed above, intrinsic cycle time and power consumption limits of SMA actuators limit actuation efficiency and speed.

Fully bidirectional legged locomotion has been developed over the past 10 years at the Italian Center for Research in Microengineering through a series of increasingly sophisticated legged robot prototypes. The first prototypes included six SMA-actuated legs [71]. The short functional lives of the SMA actuators and mechanical complexity of the design prompted a move to the use of miniature brushless DC motors, and designs incorporating 4 legs (12 $\mathrm{mm}$ in diameter and $40 \mathrm{~mm}$ in length) [72], 8 legs (12 $\mathrm{mm}$ in diameter and $40 \mathrm{~mm}$ in length) $[73,74]$, and 12 legs (11 $\mathrm{mm}$ in diameter and $25 \mathrm{~mm}$ in length) $[\mathbf{1 0}, 75]$ have been developed. This latest design (see Fig. 7(centre)) contains all mechanical components in a capsule matching the size of commercial camera pills, is able to distend tissue in a uniform manner with six points of contact at each end of the capsule (enhancing camera visibility and locomotion), and has the ability to travel the length of the colon in a time comparable to traditional colonoscopy [10]. All of the above legged designs benefit from optimized, bio-inspired leg geometry, which has included flexure-based knees and careful consideration of foot geometry for safe and high-friction contact with the intestinal wall $[\mathbf{7 6}, 77]$. Some advantages of legged locomotion include the ability to control capsule position and orientation, anchor and release the capsule as desired, and distend the colon away from the camera lens without insufflation.

A promising new approach for locomotion in a liquid-filled GI environment (particularly the stomach; the patient can be instructed to drink liquid prior to swallowing the capsule) is propeller propulsion [21], as shown in Fig. 7(right). A propeller-driven capsule acts as a miniature submarine, steering itself in a liquid environment by actuating its propellers according to direction, speed, and rotation commands input by a human operator using a joystick. The velocity of initial prototypes using this approach was $120-140 \mathrm{~cm} / \mathrm{min}$, but they do require full immersion in a liquid environment in order to work. Other innovative methods of locomotion (see reference [41]) include electrostimulation of GI muscles [78-80] and flagellar swimming [81]. In addition, some patents allude to the possibility of locomotion through magnetically actuated propellers [82], and self-propulsion through a fluid jet [83], but these mechanisms have yet to be studied in depth.

\subsubsection{Internal locomotion summary and challenges}

While, as described above, many innovative mechanisms for onboard capsule locomotion have been devised, there remain a number of challenges in moving them from research laboratories to the clinical setting. These include (a) making the mechanisms cheap enough to be disposable (which may be preferred) or robust and well-sealed enough for sterilization, (b) making them efficient enough that they can run for a
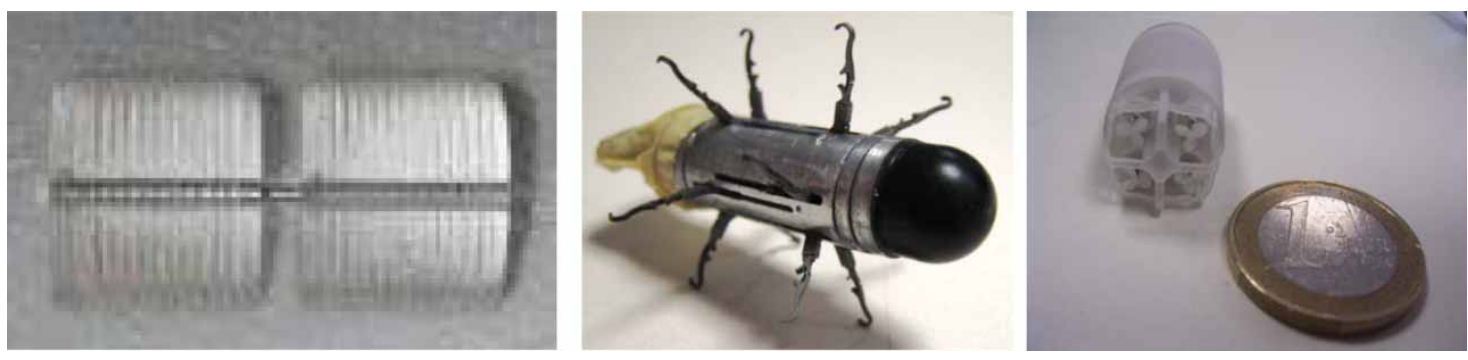

Fig. 7 Photos of various capsules that utilize internal locomotion: (left) an earthworm-like capsule that uses SMA actuation for extension and contraction (@2005 IEEE) [65], (centre) the 12-legged capsule developed by Valdastri et al. $[\mathbf{1 0 , 7 5}]$, (right) a propeller-driven capsule that acts like a miniature submarine in the stomach [21] 
sufficient length of time on miniature batteries (for example, see reference $[\mathbf{1 0}]$ for a discussion of the power requirements of the dual brushless motorlegged design and associated electronics and image capture equipment), (c) making complex mechanisms small enough to fit within a swallowable package (many existing initial prototypes have been larger than this for proof of concept), and (d) integrating locomotion mechanisms with the other modules described in Fig. 3, while maintaining overall dimensions at a swallowable size. Some of these issues are addressed, albeit at the cost of a significantly more complex overall system, by the external locomotion techniques discussed in the next section.

\subsection{External locomotion}

The primary approach to imparting forces and torques to a capsule through a system external to the patient has been through the use of magnetic fields. Magnetic actuation of internal devices has previously been demonstrated in guiding catheters (e.g. the Niobe system of Stereotaxis, Inc.) [84] and in wireless microrobots designed for use in the human eye $[\mathbf{8 5}, \mathbf{8 6}]$ and in capillaries [87].

The main advantage of using magnetic fields to control capsule position and orientation is the reduction in onboard space requirements for actuators, actuation mechanisms, and batteries. Primary challenges include determining the direction and strength of the magnetic field that should be applied to achieve desired capsule forces and torques, and dynamically modeling the capsule and intestine sufficiently well to enable control of positions and velocities via applied forces and torques. A trade-off in magnetic system design is the choice of coils versus permanent magnets for field generation. Coils can provide better control of field strength and direction, whereas permanent magnets can generate higher fields in a smaller form factor. Another critical limitation of external magnetic locomotion is that it is challenging to implement in a deflated lumen, although wireless insufflation systems have been proposed to overcome this obstacle [88].

Owing to the potential advantages, there are a number of current efforts underway to create a magnetically guided capsule. The Norika Project Team was one of the earliest groups to utilize magnetic fields for capsule motion, when they developed a capsule that has three internal coils that interact with three external coils embedded in a jacket worn by the patient for capsule rotation $[\mathbf{1 2}, \mathbf{8 9}]$. In any such system, optimization of the external magnetic field [86] is necessary to control the capsule, and approaches have been developed using three orthogonal coil pairs [90], which can be placed around the abdomen. Olympus has also been developing a magnetically actuated capsule that has an internal permanent magnet that interacts with a

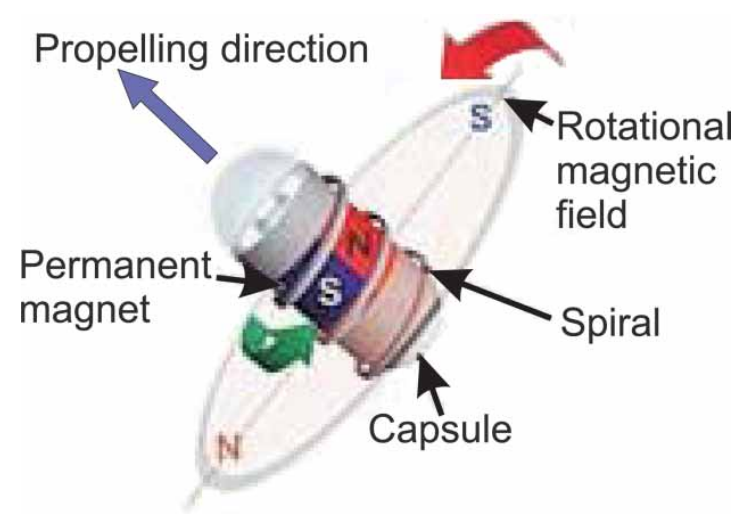

Fig. 8 Olympus magnetic capsule concept. The capsule is propelled by a rotating magnetic field that causes a helical ridge on the outside of the capsule to interact with the intestinal wall

varying, controllable, rotating field created by three pairs of external electromagnets (see Fig. 8). Rotating the external field, the capsule can be propelled forward or backward using a spiral ridge wrapped around its body [12].

Given Imaging, Inc., also has research underway towards developing a magnetic actuation system as part of a European FP6 project called Nanobased Capsule-Endoscopy with Molecular Imaging and Optical Biopsy [91]. As an alternative to external field generation using coils, Given Imaging is using an external hand-held plate magnet to generate the magnetic field. In initial feasibility studies, Given Imaging capsules have been modified to include magnetic material and an external permanent magnet was used to manipulate the capsule under gastroscopic visualization [91]. The Korean Institute of Science and Technology has proposed to control the magnetic field using an external permanent magnet fixed on the end effector of a Cartesian robot [92]. The system has a total of six degrees of freedom (DOFs): three from the robot, two from a rotary joint unit that rotates the magnet, and one from the adjustable, rolling bed of the patient. This idea has been studied in more depth by Ciuti et al. [31], who developed a user interface for control of a six-DOF industrial robot with a permanent magnet fixed to its end effector, as shown in Fig. 9. This system exploits the field strength obtained by a permanent magnet together with the precise control of magnet position afforded by the robotic manipulator.

The Stereotaxis system mentioned above, which was designed for catheters, has also recently been successfully applied to endoscopic capsule locomotion [93]. However, rather than design a new capsule with internal magnets, a magnetic shell was created for existing commercial camera pills. A silicone sleeve mixed with Neodymium-Boron-Iron can be used for this purpose [94]. Although this system can be used for orienting the capsule, because it cannot apply field gradients, 


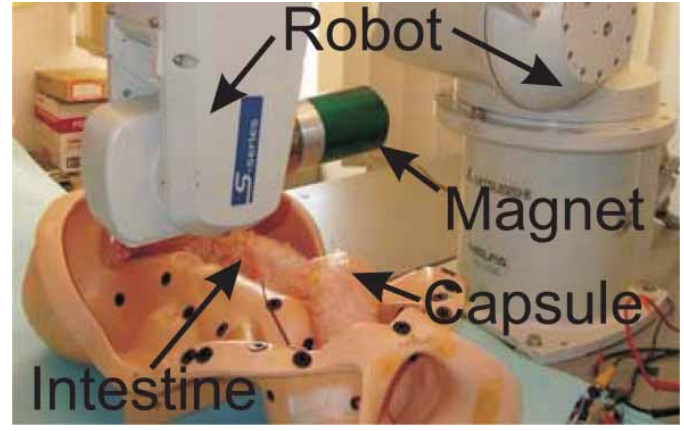

Fig. 9 An external magnet attached to the end effector of an industrial robot can be used to manoeuvre a capsule containing internal magnets through ex-vivo porcine intestine [31]

its translational capabilities are limited. Advantages of this approach are its easy integration with commercial magnetic control and endoscopic capsule systems and its low cost (assuming that the Stereotaxis system and capsule are already owned by the hospital implementing it, otherwise it would be rather expensive). Proof-of-concept experiments support the feasibility of such a system and suggest further work in shell optimization [93].

\subsubsection{External magnetic locomotion summary and challenges}

As discussed above, the primary challenges in external magnetic locomotion involve adequately controlling the magnetic field and producing locomotion in deflated portions of the GI tract. Furthermore, one intrinsic drawback to the approach is the complexity and size of the equipment needed to generate the

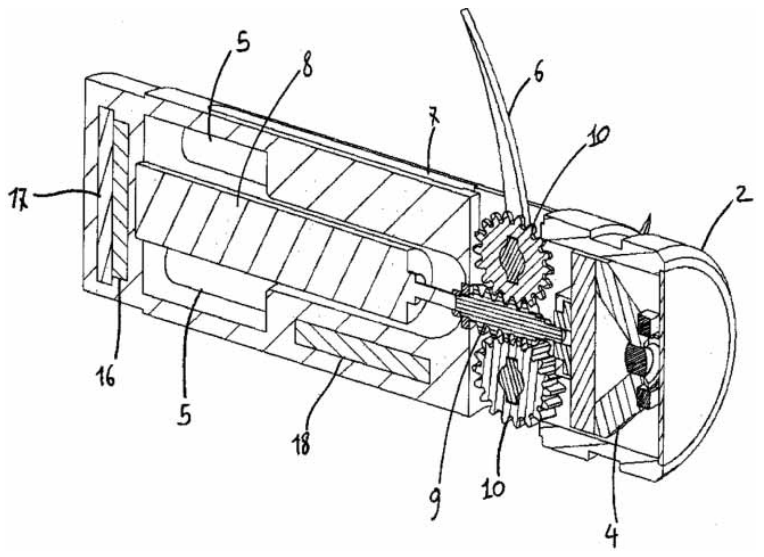

Fig. 10 Image from a recent patent of a design for a hybrid capsule that includes internal magnets and a single set of three legs actuated by a DC motor. This robot is designed to be primarily actuated via magnetic fields, with the legs used to assist when needed in tissue distention and capsule locomotion [95] magnetic field. Such equipment may involve significant additional financial costs, which will likely require specialized hospital facilities, and precludes the possibility of using capsules in small clinics and doctor's offices away from the hospital setting. However, the compelling advantage of the external magnetic locomotion approach is that it reduces or removes the need for batteries, actuators, and locomotion mechanisms onboard the capsule itself, where space is at a premium. One path forward may be a hybrid internal-external strategy with the aim of combining the benefits of both approaches. An example of such a system is described in a recent patent [95] (see Fig. 10), which is primarily magnetically actuated, but uses legs to assist with capsule motion, and orientation, and tissue distention when needed.

\section{INTERVENTION AND TISSUE MANIPULATION}

A significant limitation of modern commercial capsules is that they are strictly diagnostic devices and cannot treat the lesions they encounter directly. Thus, with more severe lesions (or for more thorough diagnosis by, e.g. biopsy), it can be necessary to perform subsequent surgery. This is why localization of capsules during image capture is currently so important (see section 3). While as reviewed in previous sections, many advancements have been made in capsule visualization and diagnosis, the interventional capabilities of capsules are comparatively in their infancy. However, some interventional capabilities have been demonstrated on proof-of-concept prototypes, including topical drug delivery, clip deployment to stop bleeding, and biopsy sampling.

A wireless endoscopic capsule for clip deployment developed by Valdastri et al. [96] is the first therapeutic capsule of its kind and has been demonstrated in in-vivo animal trials [96]. This capsule, shown in Fig. 11, has four internal permanent magnets enabling external locomotion via magnetic fields. The capsule contains a single pre-loaded SMA clip at one end, which can be fired by the action of a miniature motor housed inside the capsule, based on a wireless command issued by the human operator.

Another therapeutic capsule that can deliver topical drugs within the intestine is the iPill, under development by Philips, Inc. $[\mathbf{8}, \mathbf{5 8}]$. This capsule, which does not include a camera (also discussed briefly in section 6) aims to take internal measurements such as temperature and $\mathrm{pH}$ levels, wirelessly transmit the data to an external receiver, and deliver a topical treatment agent on command. It is hoped that the ability to deliver drugs directly at the source of the problem will lower the dosage levels and thus reduce unwanted side effects. Applications envisioned include treating colitis, Crohn's disease, and cancer $[\mathbf{8}, \mathbf{5 8}]$. Other drug delivery capsules (see also reference [13] and [33] for 

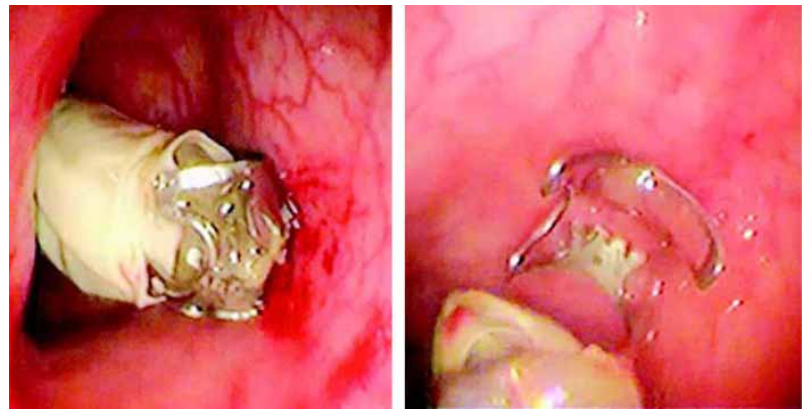

Fig. 11 Clip deployment capsule of Valdastri et al. (C2008 Georg Thieme Verlag KG) [96]. (Left) The capsule is oriented toward the lesion using external magnetic locomotion. (Right) At the command of the human operator a wireless signal triggers clip deployment, clamping the lesion and stopping bleeding

good discussions on this topic) include the InteliSite (Innovative Devices) capsule that uses SMA wires to line up perforated inner and outer sleeves to disperse a drug through the holes [33], and the Enterion capsule (Phaeton Research and Pharmaceutical Profiles) that can deliver a treating agent (liquid, powder, semisolid) using a piston/spring actuation system [33].

A biopsy device consisting of a rotational razor for cutting tissue attached to a torsion spring has also been implemented onboard a capsule platform [97]. This device, tested ex-vivo in animal intestine, was successful in extracting a tissue sample. However, it is not yet clear whether such a mechanism will be able to collect a sufficient number or volume of samples for accurate external histological analysis. Some additional ideas for intervention and tissue interaction have also recently been the subject of patent disclosures [34], and one concept consists of a dual capsule camera-biopsy system, where the two capsules are connected by a tether [98]. Capsules housing ultrasonic transducer modules have also been sketched in patents by Miyake [99] and Taniguchi [100].

\subsection{Intervention and tissue manipulation summary and challenges}

Interventional capabilities are one of the most promising areas of endoscopic capsule research. Developing a system that can see, diagnose, and treat lesions it finds is compelling from the perspectives of reducing invasiveness for the patient and reducing the financial costs of treating GI diseases. Endowing capsules with interventional capabilities is challenging due to the intelligence and miniature mechanisms required in diagnosis and treatment. However, providing clinical capsules with the interventional capabilities mentioned above or others yet to be developed has the potential to make WCEs a much more powerful weapon with which physicians can combat disease.

\section{POWER}

Since nearly every other module relies on it, the power source is a critical issue for robotic endoscopic capsules. Commercial endoscopic capsules rely on silver-oxide watch batteries, which are the only batteries of their kind approved for clinical use [1]. These batteries provide $3 \mathrm{~V}$ at $55 \mathrm{~mA}-\mathrm{hr}$ for approximately $8 \mathrm{~h}$, which implies an average power delivery of approximately $20 \mathrm{~mW}$ [101]. However, these traditional batteries are neither energy dense enough nor able to source the peak currents needed to power robotic capsules-particularly those with internal locomotion capabilities.

In general, large-scale battery technologies do not scale down well to capsule dimensions [88]. One possible exception to this rule are Lithium Ion Polymer batteries (LiPo), such as those available from Plantraco and PowerStream, which are close to the size needed for capsule integration. LiPo batteries have the highest energy density (approximately $200 \mathrm{Wh} / \mathrm{kg}$ ) available in off-the-shelf batteries, are capable of supplying peak currents up to 20 times their nominal current [41], and are miniaturizable, making them good candidates for a robotic endoscopic capsule power source.

Valdastri et al. elaborate on the power requirements of their legged capsule design, noting that the average power required for their 12-legged capsule was measured to be $430 \mathrm{~mW}$, plus an additional $180 \mathrm{~mW}$ for the real-time vision system incorporated on their prior prototypes. They come to the conclusion that a $100 \mathrm{~mA}$-h battery would be sufficient for powering their robotic capsule that is actuated by two brushless DC motors. The smallest $(10 \mathrm{~mm}$ diameter and $30 \mathrm{~mm}$ length), battery they were able to identify providing a capacity above $100 \mathrm{~mA}-\mathrm{h}$ is the TLM-1030 from Tadiran, Israel [10]. Thus, advancements in battery technology are clearly needed, together with capsule mechanism efficiency enhancements. Note, however, that it is in principle possible to manufacture customshaped LiPo batteries, so that it may be possible to shape the battery to fill whatever space is available within a capsule prototype. Thus, while it is not impossible to include batteries onboard robotic WCEs, it is clear that alternative methods of power would also be valuable, and some are being developed.

Inductive coupling provides a means of wirelessly transferring power to a capsule and is used in the intracorporal video probe [102]. This technique utilizes three internal coils onboard the capsule, which derive power from a magnetic field established by an external solenoid coil (see Fig. 12). This system is capable of providing $150 \mathrm{~mW}$ of power at any time while also remaining below the specific absorption rate limit of 


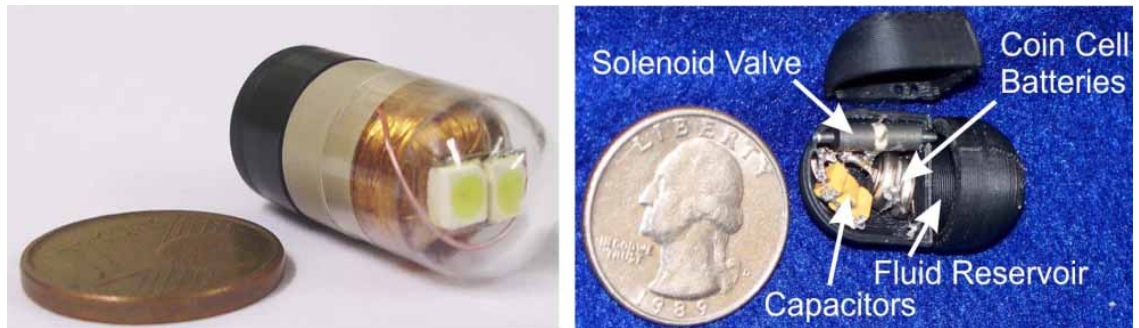

Fig. 12 (Left) Prototype capsule developed by Lenaerts et al. (@2007 Elsevier) [102], which is capable of transmitting $150 \mathrm{~mW}$ through inductive coupling. (Right) The prototype fluid-powered capsule of Toennies and Webster [88]

the patient's body. Carta et al. [103] extended this concept to power a propeller-driven capsule (see also section 7). Their design achieved $200 \mathrm{~mW}$ of power transfer (compared to $100 \mathrm{~mW}$ required for their application), and demonstrated that power transfer can be enhanced by the use of a ferromagnetic core within the capsule. The NORIKA Project Team has also proposed the use of inductive coupling to power a capsule that houses a CCD imaging chip, has focus adjustment capabilities, and has a mechanism that allows for capsule rotation (see section 7). The concept is that the patient would wear a coil vest for power transmission and direction control [89]. The coil vest worn by the patient (also mentioned in section 7) is used for both power transmission and direction control.

Another potential future power source, which promises dramatic improvement in energy and power density over conventional batteries, is the use of chemofluidic phase transition to generate pneumatic pressure. This 'fluid power' concept was initially demonstrated in a prosthetic arm by Goldfarb et al., where experiments indicated that it could improve the energetic figure of merit (a metric that encompasses power source energy density, conversion efficiency, and actuator power density) by an order of magnitude [104]. It was thus concluded by Goldfarb et al. that fluid power has the potential to be the lightest possible system capable of delivering a given amount of power and energy. Based on this, Toennies andWebster [88] proposed the idea of utilizing fluid power in capsule robots, and performed initial experiments using it for wireless insufflation, as a first step towards actuating more complex mechanisms within a capsule robot. It is noted that a beneficial potential aspect of fluid power is that 'waste gas' expelled after actuation can help to insufflate the intestine, easing capsule passage and increasing the area of intestine wall viewable in each camera image.

\subsection{Power summary and challenges}

Powering endoscopic capsule robots is the single greatest challenge facing researchers developing these robots today. While current capsules using modern battery technology can achieve some of the goals their designers envision, many remain out of reach due to power limitations. For capsule robots to achieve their full potential, they will require enhanced batteries or alternative sources of power such as inductive coupling, fluid power, or other novel technologies to be developed at the scales, power levels, and current sourcing capacity necessary for powering capsule robot systems.

\section{CONCLUSIONS}

While swallowable medical devices present substantial engineering challenges, the promise of non-invasive diagnosis and intervention deep within the body provides compelling motivation for their development. The variety of possible GI environments, diagnostic and interventional objectives, actuation and sensing strategies, and human operator interfaces inspire many diverse, innovative robotic WCE approaches. Table 1 illustrates the diverse efforts in the development of robot capsules to date. This diversity of designs and medical objectives creates a number of possible paths forward in WCE research. The most likely nearterm advancement (already underway) is the inclusion on basic commercial WCE platforms of increasingly sophisticated biosensors, vision systems, and liquid topical drug delivery capabilities. Another near-term advancement already underway is in software systems to assist with identifying diagnostically useful images and automatically analysing them to suggest likely diagnoses.

In the slightly longer term, one can expect to see clinical implementation of the most successful locomotion and anchoring mechanisms derived from prototypes currently under development in research laboratories today. It is likely that progress will continue in both internal and external locomotion and anchoring mechanisms, and even possibly in novel hybrid combinations of internal and external locomotion. Fluid-powered wireless insufflation may be an enabler of these future systems, enhancing locomotion and visualization. Eventually, it is likely that fully robotic capsules will be implemented. 
Table 1 A sampling of capsule robots and the different modules they contain. No one system to date contains all possible modules described in this article. '- ' indicates that the capability either does not exist or has not yet been explicitly reported, to the best of the authors' knowledge; 'a' indicates that the capability appears to exist, but no details have been provided; RF indicates radiofrequency; telemetry may be either (U)nidirectional or (B)idirectional; sensing may be (I)nertial, (P) for $\mathrm{pH}$, (T)emperature, (O)xygen, or (E)lectrical; locomotion may be (I)nternal or (E)xternal; power may be (B)attery, (W)ireless, (M)agnetic, or (T)ethered. This table is intended to be exemplary, not comprehensive

\begin{tabular}{|c|c|c|c|c|c|c|c|c|}
\hline Literature & Commercial & Localization & Telemetry & Sensing & Locomotion & Power & Vision & Intervention \\
\hline PillCam $[\mathbf{1}, \mathbf{4 2}]$ & $\circ$ & $\mathrm{RF}$ & $\mathrm{U}$ & - & - & B & CMOS & - \\
\hline EndoCapsule $[\mathbf{1}, \mathbf{4 4}]$ & $\circ$ & $\mathrm{RF}$ & $\mathrm{U}$ & - & - & $\mathrm{B}$ & CCD & - \\
\hline MiRo $[\mathbf{1}, \mathbf{4 0}]$ & $\circ$ & $\mathrm{RF}$ & $\mathrm{U}$ & - & - & $\mathrm{B}$ & CMOS & - \\
\hline OMOM [14, 43] & $\circ$ & $\mathrm{RF}$ & $\mathrm{U}$ & - & - & B & CMOS & - \\
\hline NORIKA [12] & $\circ$ & $\mathrm{a}$ & $\mathrm{U}$ & - & $\mathrm{E}$ & $\mathrm{W}$ & CCD & - \\
\hline Bravo pH [20] & $\circ$ & Anchored & $\mathrm{U}$ & $\mathrm{P}, \mathrm{E}$ & $\mathrm{E}$ & B & - & - \\
\hline VitalSense [57] & $\circ$ & - & $\mathrm{U}$ & $\mathrm{T}$ & - & $\mathrm{B}$ & - & - \\
\hline CorTemp [57] & $\circ$ & - & $\mathrm{U}$ & $\mathrm{T}$ & - & $\mathrm{B}$ & - & - \\
\hline iPill [58] & $\circ$ & Time & $\mathrm{U}$ & $\mathrm{P}, \mathrm{T}$ & - & B & - & Drug \\
\hline InteliSite [33] & $\circ$ & Nuclear & $\mathrm{U}$ & - & - & $\mathrm{W}$ & - & Drug \\
\hline Enterion [33] & $\circ$ & Nuclear & $\mathrm{a}$ & - & - & W & - & Drug \\
\hline Magnetic [31] & - & Inertial & $\mathrm{B}$ & I & $\mathrm{E}$ & M & CMOS & - \\
\hline $\mathrm{pH}[\mathbf{1 9}]$ & - & Anchored & $\mathrm{U}$ & $\mathrm{P}, \mathrm{E}$ & $\mathrm{E}$ & $\mathrm{B}$ & - & - \\
\hline Lab on a Pill [59] & - & - & $\mathrm{U}$ & T, P, E, O & - & $\mathrm{B}$ & - & - \\
\hline Vibration $[63]$ & - & - & $\mathrm{U}$ & - & I & B & CMOS & - \\
\hline Earthworm [65] & - & - & - & - & I & $\mathrm{T}$ & - & - \\
\hline Paddling [105] & - & - & - & - & I & $\mathrm{T}$ & - & - \\
\hline Anchor $[\mathbf{1 8}]$ & - & - & - & - & I & B & - & - \\
\hline Anchor [17] & - & - & - & - & I & $\mathrm{T}$ & - & - \\
\hline Earthworm [70] & - & - & - & - & I & $\mathrm{T}$ & a & - \\
\hline Legged $[10,71,72]$ & - & - & $\mathrm{B}$ & I & I & $\mathrm{T} / \mathrm{B}$ & CMOS & - \\
\hline Propeller [21] & - & - & $\mathrm{B}$ & - & I & $\mathrm{B}$ & - & - \\
\hline Elec. Stim. $[\mathbf{8 0}]$ & - & - & $\mathrm{U}$ & - & $\mathrm{I}$ & $\mathrm{B}$ & - & - \\
\hline Magnetic [91] & - & $\mathrm{RF}$ & $\mathrm{U}$ & - & $\mathrm{E}$ & $\mathrm{B}$ & CMOS & - \\
\hline Magnetic [93] & - & $\mathrm{RF}$ & $\mathrm{U}$ & - & $\mathrm{E}$ & $\mathrm{B}$ & CMOS & - \\
\hline Clip [96] & - & - & $\mathrm{B}$ & - & $\mathrm{E}$ & $\mathrm{B}$ & - & Clip \\
\hline Biopsy [97] & - & - & - & - & - & W & - & Biopsy \\
\hline Propeller [103] & - & - & $\mathrm{B}$ & - & I & $\mathrm{W}$ & - & - \\
\hline Inflation [88] & - & - & - & - & - & $\mathrm{B}$ & - & Inflation \\
\hline Autofocus [14] & - & - & $\mathrm{B}$ & - & - & $\mathrm{B}$ & CMOS & - \\
\hline
\end{tabular}

These fully robotic devices may be autonomous, teleoperated, or some mixture of the two. Fully autonomous systems require advanced intelligence to locate, recognize, and treat the lesions they encounter without the aid of real-time human decision making. Internal locomotion appears most promising for such systems, so that they can be used in many different non-hospital settings, including being selfadministered by patients in their own homes. Significant advancements will be required in power sources and capsule intelligence to enable fully autonomous capsules.

Another very different future fully robotic capsular device that can be envisioned is a teleoperated system. A teleoperated capsule may work under the control of a human operator who inputs commands using a console similar to that of the da Vinci ${ }^{\circledR}$ system, Intuitive Surgical, Inc. Creating a teleoperated capsule robot will require advancements in nearly all modules discussed in this article. For example, image capture, compression, and transmission techniques will all have to improve to the point where real-time video can be obtained from wireless capsules. The capsule itself will also need to be instrumented with miniature manipulators, and of course, a high energy density power source. The future feasibility of including all of these systems on one capsule is currently unclear, but it is also conceptually possible to achieve such goals via cooperative teams of capsules that work together and/or assemble themselves into a larger structure. Such future systems may also move beyond the GI tract and into the abdomen in a NOTES-like manner to perform scarless surgical interventions.

Regardless of the form future systems take, it is clear that modern commercial WCEs have already proven their diagnostic worth, and that swallowable devices represent an important emerging area of medical technology. The next fundamental leap forward in capsule technology will be the inclusion of interventional capabilities onboard the capsule, and initial prototype interventional devices have recently begun to appear in research laboratories. Given the promise of capsule robots in reducing the invasiveness, indignity, financial costs, and anaesthesia-related risks in GI procedures, the outlook for innovation in swallowable devices is bright. 


\section{ACKNOWLEDGEMENTS}

This work was supported in part by a Vanderbilt University Discovery Grant, the NSF Graduate Research Fellowship Programme, and the VECTOR FP6 European Project EU/IST-2006-033970.

(C) Authors 2010

\section{REFERENCES}

1 Moglia, A., Menciassi, A., Dario, P., and Cuschieri, A. Capsule endoscopy: progress update and challenges ahead. Nat. Rev. Gastroenterol. Hepatol., 2009, 6, 353362.

2 Fantastic journey. The Economist Report, 18 April 2009, pp. 11-13.

3 Menciassi, A., Quirini, M., and Dario, P. Microrobotics for future gastrointestinal endoscopy. Minim. Invasive Therapy Allied Technol., 2007, 16, 91-100.

4 Sclabas, G., Swain, P., and Swanstrom, L. Endoluminal methods for gastrotomy closure in natural orifice transenteric surgery (NOTES). Surg. Innov., 2006, 13, 23-30.

5 Spaun G. and Swanstrom, L. Quo vaids NOTES? Eur. Sur., 2008, 40, 211-219.

6 Scott, D., Tang, S., Fernandez, R., Bergs, R., Goova, M., Zeltser, I., Kehdy, F., and Cadeddu, J. Completely transvaginal NOTES cholecystectomy using magnetically anchored instruments. Surg. Endosc., 2007, 21, 2308-2316.

7 Lehman, A., Dumpert, J., Wood, N., Redden, L., Visty, A., Farritor, S., Varnell, B., and Oleynikov, D., Natural orifice cholecystectomy using a miniature robot. Surg. Endosc., 2009, 23, 260-266.

8 Forgione, A. In vivo microrobots for natural orifice transluminal surgery. current status and future perspectives. Surg. Oncol., 2009, 18, 121-129.

9 Cotton, P. B. and Williams, C. B. Practical gastrointestinal endoscopy the fundamentals, 5th edition, 2003, (Wiley-Blackwell, Oxford).

10 Valdastri, P., Webster, III R. J., Quaglia, C., Quirini, M., Menciassi, A., and Dario, P. A new mechanism for mesoscale legged locomotion in compliant tubular environments. IEEE Trans. Robot., 2009, 25, 1047-1057.

11 Waterman, M. and Eliakim, R. Capsule enteroscopy of the small intestine. Abdom. Imaging, 2008, 34, $452-458$.

12 Moglia, A., Menciassis, A., Schurr, M., and Dario, P. Wireless capsule endoscopy: from diagnostic devices to multipurpose robotic systems. Biomed. Microdevices, 2007, 9, 235-243.

13 Twomey, K. and Marchesi, J. R. Swallowable capsule technology: current perspectives and future directions. Endoscopy, 2009, 41, 357-362.

14 Cavallotti, C., Piccigallo, M., Susilo, E., Valdastri, P., Menciassi, A., and Dario, P. An integrated vision system with autofocus for wireless capsular endoscopy. Sens. Actuators A, 2009, 156, 72-78.

15 Gossum, A. V., Navas, M. M., Fernandez-Urien, I., Carretero, C., Gay, G., Delvaux, M., Lapalus, M. G.,
Ponchon, T., Neuhaus, H., Philipper, M., Costamagna, G., Riccioni, M. E., Spada, C., Petruzziello, L., Fraser, C. Postgate, A., Fitzpatrick, A., Hagenmuller, F., Keuchel, M., Schoofs, N., and Deviere, J. Capsule endoscopy versus colonoscopy for the detection of polyps and cancer. N. Engl. J. Med., 2009, 361 (3), 264-270.

16 Encyclopedia Britannica Online. Human digestive system, 2009, available from http:/ / www.britannica.com/.

17 Glass, P., Cheung, E., and Sitti, M. A legged anchoring mechanism for capsule endoscopes using micropatterned adhesives. IEEE Trans. Biomed. Eng., 2008, 55 2759-2767.

18 Tognarelli, S., Quaglia, C., Valdastri, P., Susilo, E., Menciassi, A., and Dario, P. Innovative stopping mechanism for esophageal wireless capsular endoscopy. Procedia Chem., 2009, 1, 485-488.

19 Gonzalez-Guillaumin, J., Sadowski, D., Kaler, K., and Mintchev, M. Ingestible capsule for impedance and pH monitoring in the esophagus. IEEE Trans. Biomed. Engng, 2007, 54, 2231-2236.

20 Kwiatek, M. A. and Pandolfino, J. E. The Bravo(TM) pH capsule system. Dig. Liver Dis., 2008, 40, 156-160.

21 Tortora, G., Valdastri, P., Susilo, E., Menciassi, A., Dario, P., Rieber, F, and Schurr, M. Propeller-based wireless device for active capsular endoscopy in the gastric district. Minim. Invasive Therapy Allied Technol., 2009, 18 280-290.

22 Nagy, Z., Harada, K., Fluckiger, M., Susilo, E., Kaliakatsos, I. K., Menciassi, A., Hawkes, E., Abbott, J. J., Dario, P., and Nelson, B. J. Assembling reconfigurable endoluminal surgical systems: Opportunities and challenges. Int. J. Biomechatronics and Biomed. Robot., 2009, 1 , $3-16$.

23 Hounnou, G., Destrieux, C., Desme, J., Bertrand, P., and Velut, S. Anatomical study of the length of the human intestine. Surg. and Radiol. Anat., 2002, 24, 290-294.

24 Drossman, D. A., Grimm, I. S., and Shaheen, N. J. Handbook of gastroenterologic procedures, 4th edition, 2005 (Lippincott Williams \& Wilkins, Philadelphia, Pennsylvania).

25 Fischer, D., Schreiber, R., Levi, D., and Eliakim, R. Capsule endoscopy: the localization system. Gastrointest. Endos. Clin. N. Am., 2004, 14, 25-31.

26 Wang, X., Meng, M., and Hu, C. A localization method using 3-axis magnetoresistive sensors for tracking of capsule endoscope. In Proceedings of the IEEE/EMBS, New York, NY, USA, 2006, pp. 2522-2525.

$27 \mathrm{Hu}$, C., Meng, M., and Mandal, M. The calibration of 3-axis magnetic sensor array system for tracking wireless capsule endoscope. In Proceedings of the IEEE/RSJ International Conference on Intelligent robots and systems, Beijing, P. R. China, 2006, pp. 162-167.

28 Hocke, M., Schone, U., Richert, H., Gornert, P., Keller, J., Layer, P., and Stallmach, A. Every slow-wave impulse is associated with motor activity of the human stomach. Am. J. Physiol. Gastrointest. Liver Physiol., 2008, 296, 709-716.

29 Bulat, J., Duda, K., Duplaga, M., Fraczek, R., Skalski, A., Socha, M., Turcza, P., and Zielinski, T. Data processing tasks in wireless GI endoscopy: image-based capsule localization \& navigation with video compression. In Proceedings of the IEEE/EMBS, Lyon, France, 2007, pp. 2815-2818. 
30 Lee, J., Oh, J., Shah, S., Yuan, X., and Tang, S. Automatic classification of digestive organs in wireless endoscopy videos. In Proceedings of the ACM Symposium on Applied computing, Seoul, Rep. of Korea, 2007.

31 Ciuti, G., Valdastri, P., Menciassi, A., and Dario, P. Robotic magnetic steering and locomotion of microsystems for diagnostic and surgical endoluminal procedures. Robotica, 2010, 28(2), 199-207. DOI: $10.1017 /$ S0263574709990361.

32 Arshak, K. and Adepoju, F. Capsule tracking in the GI tract: a novel microcontroller based solution. In Proceedings of the IEEE Sensors Applications Symposium, Houston, Texas, USA, 2006, pp. 186-191.

33 Wilding, I., Hirst, P., and Connor, A. Development of a new engineering-based capsule for human drug absorption studies. Pharm. Sci. Technol. Today, 2000, 3, 385-392.

34 Moglia, A., Menciassi, A., and Dario, P. Recent patents on wireless capsule endoscopy. Recent Patents Biomed. Eng., 2008, 1, 24-33.

35 Kuth, R., Reinschke, J., and Roeckelein, R. Method for determining the position and orientation of an endoscopy capsule guided through an examination object by using a navigating magnetic field generated by means of a navigation device. DE102005032370, 2007.

36 Thone, J., Radiom, S., Turgis, D., Carta, R., Gielen, G., and Puers, R. Design of a 2 Mbps FSK near-field transmitter for wireless capsule endoscopy. Sens. Actuators $A, 2009,156,43-48$.

37 Shen, M.-W., Lee, C.-Y., and Bor, J.-C. A 4.0-mW 2-Mbps programmable BFSK transmitter for capsule endoscope applications. In Proceedings of the IEEE Asian SolidState Circuits Conference, Hsinchu, Taiwan, 2005.

38 Susilo, E., Valdastri, P., Menciassi, A., and Dario, P. A miniaturized wireless control platform for robotic capsular endoscopy using advanced pseudokernel approach. Sens. Actuators A, Phys., 2009, 156, 49-58.

39 Valdastri, P., Menciassi, A., and Dario, $\mathbf{P}$. Transmission power requirements for novel ZigBee implants in the gastrointestinal tract. IEEE Trans. Biomed. Eng., 2008, 55, 1705-1710.

40 Bang, S., Park, J., Jeong, S., Kim, Y., Shim, H., Kim, T., Lee, D., and Song, S. First clinical trial of the "MiRo" capsule endoscope by using a novel transmission technology: electric-field propagation. Clin. Endos., 2009, 69, 253-259.

41 Swain, P. The future of wireless capsule endoscopy. World J. Gastroenterol. 2008, 14, 4142-4145.

42 Given Imaging, Inc.: www.givenimaging.com.

43 Jinshan Science and Technology Group, Ltd., Co. http://www.omom.us/main.php?sLAN=en.

44 Olympus, Inc.: www.olympus.com.

45 Kim, T., Song, S., Jung, H., Kim, J., and Yoon, E. Micro capsule endoscope for gastro intestinal tract. In Proceedings of the IEEE/EMBS, Lyon, France, 2007, pp. 2823-2826.

46 Metzger, Y., Adler, S., Shitrit, A., Koslowsky, B., and Bjarnason, I. Comparison of a new PillCam SB2 video capsule versus the standard PillCam SB for detection of small bowel disease. Med. Imaging, 2009, 2, 7-11.

47 Cave, D., Fleischer, D., Gostout, C., Faigel, D., Leighton, J., Heigh, R., Sharma, V., Mergener, K., Bhattacharya, K., Rajan, E., Foley, A., Lee, M., Knipschield, M., and
Hibberd, P. A multi-center randomized comparison of the EndoCapsule: Olympus Inc and the PillCam SB: Given Imaging in patients with obscure GI bleeding [abstract]. Gastrointest. Endos., 2007, 65, AB125.

48 Vatteroni, M., Covi, D., Cavallotti, C., Valdastri, P., Menciassi, A., Dario, P., and Sartori, A. Smart optical CMOS sensor for endoluminal applications. Procedia Chem., 2009, 1, 1271-1274.

49 VECTOR: www.vector-project.com.

50 Hasan, M. and Wallace, M. Image-enhanced endoscopy. Am. Soc. Gastrointest. Endos., 2009, 16, 1-5.

51 Turgis, D. and Puers, R. Image compression in video radio transmission for capsule endoscopy. Sens. Actuators $A, 2005,123-124,129-136$

52 Lin, M.-C., Dung, L.-R., and Weng, P.-K. An ultralow-power image compressor for capsule endoscope. BioMed. Eng. OnLine, 2006, 5, 14.

53 Turcza, P., Zielinski, T., and Duplaga, M. Hardware implementation aspects of new low complexity image coding algorithm for wireless capsule endoscopy. Int. Conf. Computat. Sci., 2008, 5101, 476-485.

54 Zabulis, X., Argyros, A., and Tsakiris, D. Lumen detection for capsule endoscopy. In Proceedings of the IEEE/RSJ International Conference on Intelligent robots and systems, Nice, France, 2008, pp. 3921-3926.

55 Wang, X. and Meng, M. In situ analysis of capsule endoscopy images. In Proceedings of the IEEE/RSJ International Conference on Intelligent robots and systems, St Louis, Missouri, USA, 2009.

56 Bejakovic, S., Kumar, R., Dassopoulos, T., Mullin, G., and Hager, G. Analysis of crohn's disease lesions in capsule endoscopy images. In Proceedings of the IEEE International Conference on robotics and automation, Kobe, Japan, 2009.

57 McKenzie, J. and Osgood, D. Validation of a new telemetric core temperature monitor. J. Therm. Biol., 2004, 29, 605-611.

58 Philips, Inc.: www.philips.com.

59 Johannessen, E. A., Wang, L., Cui, L., Tang, T. B., Ahmadian, M., Astaras, A. Reid, S. W. J., Yam, P. S., Murray, A. F., Flynn, B. W., Beaumont, S. P., Cumming, D. R. S., and Cooper, J. M. Implementation of multichannel sensors for remote biomedical measurements in a microsystems format. IEEE Trans. Biomed. Eng., 2004, 51, 525-535.

60 Allison, E., Kiraly, Z., Springer, G., and Dam, J. Endocapsule. Patent number WO/2006/045011, 2006.

61 Wang, T. D. and Van Dam, J. Optical biopsy: a new frontier in endoscopic detection and diagnosis. Clin. Gastroenterol. Hepatol., 2004, 2, 744-753.

62 Westerhof, J., Weersman, R., and Koornstra, J. Risk factors for incomplete small-bowel capsule endoscopy. Gastrointest. Endos., 2009, 69, 74-80.

63 Zabulis, X., Sfakiotakis, M., and Tsakiris, D. Effects of vibratory actuation on endoscopic capsule vision. In Proceedings of the IEEE/EMBS Conference, Vancouver, British Columbia, Canada, 2008, pp. 5901-5904.

64 Kim, B., Lee, S., Park, J. H., and Park, J.-O. Design and fabrication of a locomotive mechanism for capsuletype endoscopes using shape memory alloys (SMAs). IEEE Trans. Mechatronics, 2005, 10, 77-86.

65 Kim, B., Park, S., Yoon, S.-J., and Jee, C. Y. An earthworm-like locomotive mechanism for capsule 
endoscopes. In Proceedings of the IEEE/RSJ International Conference on Intelligent robots and systems, Edmonton, Alberta, Canada, 2005, pp. 2997-3002.

66 Kwon, J., Park, S., Park, J., and Kim, B. Evaluation of the critical stroke of an earthworm-like robot for capsule endoscopes. Proc. IMechE, Part H: J. Engineering in Medicine, 2007, 221, 397-405. DOI: 10.1243/09544119JEIM134.

67 Li, W, Guo, W., Li, M., and Zhu, Y. A novel locomotion principle for endoscopic robot. In Proceedings of the IEEE International Conference on Mechatronics and automation, Luoyang, P. R. China, 2006, pp. 1658-1662.

68 Feng,Y., Li, W., Li, M., and Sun, L. Structure optimization of the endoscopic robot ciliary leg based on dimensional analysis. In Proceedings of the IEEE International Conference on Robotics and biomimetics, Sanya, P. R. China, 2007, pp. 109-114.

69 Park, S., Park, H., Park, S., and Kim, B. A paddling based locomotive mechanism for capsule endoscopes. J. Mech. Sci. Technol., 2006, 20, 1012-1018.

70 Karagozler, M., Cheung, E., Kwon, J., and Sitti, M. Miniature endoscopic capsule robot using biomimetic micro-patterned adhesives. In Proceedings of the IEEE/RAS-EMBS International Conference on Biomedical robotics and biomechatronics, Pisa, Italy, 2006, pp. 105-111.

71 Gorini, S., Quirini, M., Menciassi, A., Pernorio, G., Stefanini, C., and Dario, P. A novel SMA-based actuator for a legged endoscopic capsule. In Proceedings of the IEEE/RAS-EMBS International Conference on Biomedical robotics and biomechatronics, Pisa, Italy, 2006, pp. 443-449.

72 Quirini, M., Menciassi, A., Scapellato, S., Stefanini, C., and Dario, P. Design and fabrication of a motor legged capsule for the active exploration of the gastrointestinal tract. IEEE/ASME Trans. Mechatronics, 2008, 13, 169179.

73 Quirini, M., Scapellato, S., Valdastri, P., Menciassi, A., and Dario, P. An approach to capsular endoscopy with active motion. In Proceedings of the IEEE/EMBS, Lyon, France, 2007, pp. 2827-2830.

74 Quirini, M., Menciassi, A., Scapellato, S., Dario, P., Rieber, F., Ho, C-N., Schostek, S., and Schurr, M. Feasibility proof of a legged locomotion capsule for the GI tract. Gastrointest. Endos., 2008, 67, 1153-1158.

75 Quaglia, C., Buselli, E., Webster, III R. J., Valdastri, P., Menciassi, A., and Dario, P. An endoscopic capsule robot: a meso-scale engineering case study. $J$. Micromech. Microeng., 2009, 19, 105007.

76 Buselli, E., Valdastri, P., Quirini, M., Menciassi, A., and Dario, P. Superelastic leg design optimization for an endoscopic capsule with active locomotion. Smart Mater. Struct., 2009, 18, 015001 (8pp).

77 Buselli, E., Pensabene, V., Castrataro, P., Valdastri, P. Menciassi, A., and Dario, P. Micro hairs for friction enhancement in active capsular endoscopy. In Proceedings of the Second European Conference on Tribology, Pisa, Italy, 2009, pp. 25-30.

78 Mosse, A., Mills, T., Appleyard, M., Kadirkamanathan, S., and Swain, P. Electrical stimulation for propelling endoscopes. Gastrointest. Endos., 2001, 54, 79-83.

79 Woo, S. H., Jang, J. Y., Jung, E. S., Lee, J. H., Moon Y. K., Kim, T. W., Won, C. H., Choi, H. C., and Cho, J. H.
Electrical stimuli capsule for control moving direction at the small intestine. In Proceedings of the IASTED International Conference on Biomedical engineering, Innsbruck, Austria, 2006.

80 Woo, S. H., Kim, T. W., Lee, J. H., Kim, P. U., Won, C. H., and Cho, J. H. Implemented edge shape of an electrical stimulus capsule. Int. J. Med. Robot. Comput. Assist. Surg., 2009, 5, 59-65.

81 Kosa, G., Jakb, P., Hata, N., Jolesz, F., Neubach, Z., Shoham, M. Zaaroor, M., and Szekely, G. Flagellar swimming for medical micro robots: Theory, experiments, and application. In Proceedings of the IEEE/RAS-EMBS International Conference on Biomedical robotics and biomechatronics, Scottsdale, Arizona, USA, 2008, pp. 258-263.

82 Iddan, G. and Gilad, Z. Motor for an in-vivo device. Patent number WO 2006/070350 A2, 2006.

83 Iddan, G. Self propelled device. US Patent number 2006/0030754 A1, 2006.

84 Ramcharitar, S., Patterson, M. S., Geuns, R. J., Meighem, C., and Serruys, P. W. Technology insight: magnetic navigation in coronary interventions. Nat. Clin. Pract. Cardiovasc. Med., 2008, 5, 148-156.

85 Ergeneman, O., Dogangil, G., Kummer, M. P., Abbott, J. J., Nazeeruddin, M. K., and Nelson, B. J. A magnetically controlled wireless optical oxygen sensor for intraocular measurements. IEEE Sen. J. Am. Chem. Soc., 2008, 8, 29-37.

86 Abbott, J. J., Ergeneman, O., Kummer, M. P., Hirt, A.M. and Nelson, B. J. Modeling magnetic torque and force for controlled manipulation of soft-magnetic bodies. IEEE Trans. Robot., 2007, 23, 1247-1252.

87 Martel, S., Felfoul, O., Mathieu, J.-B., Chanu, A., Tamaz, S., Mohammadi, M., Mankiewicz, M., and Tabatabaei, N. MRI-based medical nanorobotic platform for the control of magnetic nanoparticles and flagellated bacteria for target interventions in human capillaries. Int. J. Robot. Res., 2009, 28, 1169-1182.

88 Toennies, J. L. and Webster, III R. J. A wireless insufflation system for capsular endoscopes. ASME J. Med. Devices, 2009, 3(2), 27514.

89 Uehara, A. and Hoshina, K. Capsule endoscope norika system. Minim. Invasive Therapy Allied Technol., 2003, $1,227-234$

90 Wang, X. and Meng, M. A magnetic stereo actuation mechanism for active capsule endoscope. In Proceedings of IEEE/EMBS, Lyon, France, 2007, pp. 2811-2814.

91 Volke, F., Keller, J., Schneider, A., Gerber, J., ReimannZawadzki, M., Rabinovitz, B., Mosse, C., and Swain, P. In-vivo remote manipulation of modified capsule endoscopes using an external magnetic field [abstract]. Gastrointest. Endos., 2008, 67, AB121-AB122.

92 Kim, B. K., Park, J. O., and Hong, Y. S. Capsule type endoscope control system. Patent Number WO/2005/122866, Korean Institute of Science and Technology, 2005.

93 Carpi, F. and Pappone, C. Magnetic maneuvering of endoscopic capsules by means of a robotic navigation system. IEEE Trans. Biomed. Eng., 2009, 56, 1482-1490.

94 Carpi, F., Galbiati, S., and Carpi, A. Magnetic shells for gastrointestinal endoscopic capsules as a means to control their motion. Biomed. Pharmacother., 2006, 60 370-374. 
95 Menciassi, A., Dario, P., Valdastri, P., Quaglia, C., and Buselli, E. Hybrid active locomotion teleoperated endoscopic capsule. International patent application number PCT/IB2009/054491, 2009.

96 Valdastri, P., Quaglia, C., Susilo, E., Menciassi, A., Dario, P., Ho, C. N., Anhoeck, G., and Schurr, M. O. Wireless therapeutic endoscopic capsule: in vivo experiment. Endoscopy, 2008, 40, 979-982.

97 Kong, K., Cha, J., Jeon, D., and Cho, D. A rotational micro biopsy device for the capsule endoscope. In Proceedings of the IEEE/RSJ International Conference on Intelligent robots and systems, Edmonton, Alberta, Canada, 2005, pp. 1839-1843.

98 Swain, C. P. Method, system and device for in-vivo biopsy. Patent number EP1757099, 2007.

99 Miyake, K. Capsule ultrasonic endoscope and capsule ultrasonic endoscope system. Patent number JP325874, 2006.

100 Taniguchi, Y. Intracoelomic mobile body, and capsule-type ultrasonic endoscope. Patent number US20050119577, 2005.
101 Carey, D. Camera-in-a-pill takes pictures of digestive tract, August 2006. Optical Electronics and Display, available from http://www.eetasia.com/ ART_8800430920_480700_NP_6342156c.HTM.

102 Lenaerts, B. and Puers, R. An inductive power link for a wireless endoscope. Biosens. Bioelectron., 2007, 22, 1390-1395.

103 Carta, R., Tortora, G., Lenaerts, B., Thone, J., Valdastri, P., Menciassi, A., Puers, R., and Dario, P. Wireless powering for a self-propelled and steerable endoscopic capsule for stomach inspection. Biosens. Bioelectron, 2009, 25, 845-851.

104 Goldfarb, M., Barth, E., Gongola, M., and Wehrmeyer, J., Design and energetic characterization of a liquidpropellant-powered actuator for self-powered robots. IEEE/ASME Trans. Mechatronics, 2003, 8, 254-262.

105 Park, H., Park, S., Yoon, E., Kim, B., Park, J., and Park, S. Paddling based microrobot for capsule endoscopes. In Proceedings of the IEEE International Conference on Robotics and automation, Roma, Italy, 2007, pp. 33773382. 